\section{Tocilizumab for the treatment of polyarteritis nodosa: a systematic literature review}

We read the paper by Rimar et $a l^{1}$ in your journal with great interest. They reported the case with refractory polyarteritis nodosa treated with tofacitinib, a janus kinase inhibitor, successfully. As shown in their paper, recent advances in the era of biologic agents have improved the management of difficult-to-treat cases dramatically. Considering that tofacitinib blocks interleukin (IL)-6-mediated signalling pathway through inhibiting janus kinase 1, inhibiting IL-6 cascade may also be effective in polyarteritis nodosa. In this regard, tocilizumab, a biologic agent targeting IL-6 receptor, has shown its efficacy in a variety of diseases such as rheumatoid arthritis, adult-onset Still's disease, large-vessel vasculitis and Behcet's disease. ${ }^{2-5}$ Although the precise pathogenesis of polyarteritis nodosa remains unclear, serum IL-6 levels correlate with disease severity, suggesting the involvement of IL- 6 in the disease process. ${ }^{6}$ Therefore, we assume that tocilizumab may benefit polyarteritis nodosa as a therapeutic option.

To investigate the effectiveness and safety profile of tocilizumab in patients with polyarteritis nodosa, we performed a systematic literature review from the inception dates until 23 July 2020. We used the PubMed database to identify all English publications using the Medical Subject Heading 'polyarteritis nodosa' and 'tocilizumab' and identified 13 potentially relevant articles. Among them, seven were excluded due to the following: four reviews, two duplicates and one non-related topic. Eventually, a total of 11 cases with polyarteritis nodosa treated with tocilizumab were identified from six articles (table 1). ${ }^{7-12}$ The median age of the cases was 35 years old (range: $3-70$ ), with equal sex distribution. The median disease duration at tocilizumab treatment was 38 months (range: 3-120). Clinical symptoms varied through the cases (online supplementary table 1). All patients showed high levels of serum $\mathrm{C}$ reactive protein (median, $19.7 \mathrm{mg} / \mathrm{dL}$ ). As shown in table 1 , the reasons for initiating tocilizumab were the following: nine cases with refractory to and/or relapsing clinical course by prior immunosuppressive treatments such as cyclophosphamide $(n=6)$, methotrexate $(n=4)$, mycophenolate mofetil $(n=2)$, azathioprine $(n=2)$, tacrolimus $(n=1)$, anti-tumour necrosis factor agents $(n=2)$, rituximab $(n=1)$ and anakinra $(\mathrm{n}=1)$. In the other two cases, tocilizumab was initiated as a primary induction therapy. Tocilizumab was used as the intravenous administration at a dose of $8 \mathrm{mg} / \mathrm{kg}$ every 4 weeks in seven cases and every 2 weeks in one case, at a dose of 10 $\mathrm{mg} / \mathrm{kg}$ every 4 weeks in one case and subcutaneous administration at a dose of $162 \mathrm{mg}$ weekly in two cases. In seven cases, tocilizumab was used in combination with high-dose glucocorticoids (table 1). The median observation period after tocilizumab treatment was 12 months (range: 6-37). In all cases, tocilizumab rapidly improved clinical manifestations, mostly within a week, and glucocorticoids could be successfully tapered. All cases achieved asymptomatic condition at last visit. Glucocorticoids were completely stopped in three cases, while eight cases were receiving only low dose ( $\leq 5 \mathrm{mg} /$ day) at last visit (table 1$)$. No new safety signal or adverse event was reported.

As this literature review was based on case reports, in which positive results are inclined to be published, potential publication bias can exist. Further, the number of case reports was small due to the rarity of the disease. Nevertheless, tocilizumab is effective in cases of refractory/relapsing polyarteritis nodosa and showed its glucocorticoid-sparing effect. It could even achieve glucocorticoid-free in some cases. Generally, the prognosis of polyarteritis nodosa is poor, showing the 5 -year survival rate as $13 \%$ if untreated and as $80 \%$ even if treated..$^{13}$ Our study along with the one by Rimar et al would shed light on the management of this rare disease by biologic agents to improve their prognosis. Future prospective randomised controlled trials are desired to confirm our results.

\section{Mitsuhiro Akiyama $\odot$, Yuko Kaneko, Tsutomu Takeuchi}

Division of Rheumatology, Department of Internal Medicine, Keio University Shinanomachi Campus, Shinjuku-ku, Tokyo, Japan

Correspondence to Dr Yuko Kaneko, Division of Rheumatology, Department of Internal Medicine, Keio University School of Medicine, 35 Shinanomachi, Shinjuku-ku, Tokyo, Japan; ykaneko.z6@keio.jp

\begin{tabular}{|c|c|c|c|c|c|c|c|c|c|c|}
\hline No & $\begin{array}{l}\text { Age } \\
\text { (years) }\end{array}$ & Sex & $\begin{array}{l}\text { Disease } \\
\text { duration } \\
\text { (months) }\end{array}$ & $\begin{array}{l}\text { Treatments before } \\
\text { TCZ }\end{array}$ & CRP $(\mathrm{mg} / \mathrm{dL})$ & $\begin{array}{l}\text { The } \\
\text { reason for } \\
\text { TCZ }\end{array}$ & Dose/frequency & $\begin{array}{l}\text { Concomitant } \\
\text { treatment }\end{array}$ & $\begin{array}{l}\text { Observation } \\
\text { (months) }\end{array}$ & $\begin{array}{l}\text { PSL at last } \\
\text { visit }\end{array}$ \\
\hline 1 & 11 & $\mathrm{~F}$ & 43 & $\begin{array}{l}\text { GC, AZA, MMF, TAC, } \\
\text { CyA, Ivlg, ETN, IFX, } \\
\text { ADA }\end{array}$ & Elevated & $\mathrm{R}$ & $8 \mathrm{mg} / \mathrm{kg}$ IV every 2 weeks & PSL, MMF & 7 & Off \\
\hline 2 & 23 & M & 38 & $\begin{array}{l}\text { GC, CYC, MTX, RTX, } \\
\text { ANA, Ivlg }\end{array}$ & 29.1 & $\mathrm{R}$ & 8 mg/kg IV every 4 weeks & PSL 80 mg/day & 37 & 4 mg/day \\
\hline 3 & 24 & $M$ & NA & GC, CYC, Ivlg & 29.8 & $\mathrm{R}$ & $8 \mathrm{mg} / \mathrm{kg}$ IV every 4 weeks & mPSL 250 mg IV & 11 & $5 \mathrm{mg} /$ day \\
\hline 4 & 63 & $\mathrm{~F}$ & NA & GC & 17.4 & $P$ & 162 mg SC every week & PSL 50 mg/day & 6 & $5 \mathrm{mg} /$ day \\
\hline 5 & 70 & $\mathrm{~F}$ & NA & GC, MTX & 9.3 & $\mathrm{R}$ & 8 mg/kg IV every 4 weeks & mPSL 500 mg IV & 13 & $5 \mathrm{mg} /$ day \\
\hline 6 & 67 & $M$ & 6 & $\mathrm{GC}, \mathrm{CYC}$ & 2.03 & $\mathrm{R}$ & 162 mg SC every week & PSL 16 mg/day, MTX & 15 & 4 mg/day \\
\hline 7 & 39 & $\mathrm{~F}$ & 120 & $\begin{array}{l}\text { GC, CYC, MTX, MMF, } \\
\text { IFX }\end{array}$ & $5.9-12.6$ & $\mathrm{R}$ & $8 \mathrm{mg} / \mathrm{kg}$ IV every 4 weeks & PSL 50 mg/day & 12 & $5 \mathrm{mg} /$ day \\
\hline 8 & 52 & $\mathrm{~F}$ & 96 & $\begin{array}{l}\text { GC, CYC, MTX, AZA, } \\
\text { dapsone }\end{array}$ & Not mentioned & $\mathrm{R}$ & 8 mg/kg IV every 4 weeks & PSL 35 mg/day & 12 & $5 \mathrm{mg} /$ day \\
\hline 9 & 35 & $M$ & 3 & GC, Ivlg & 39.3 & $\mathrm{R}$ & $8 \mathrm{mg} / \mathrm{kg}$ IV every 4 weeks & PSL 60 mg/day & 10 & Off \\
\hline 10 & 33 & M & NA & $\mathrm{GC}, \mathrm{CYC}$ & 16.9 & $\mathrm{R}$ & 8 mg/kg IV every 4 weeks & PSL 4 mg/day & 50 & Off \\
\hline 11 & 3 & M & 9 & None & 21.9 & $\mathrm{P}$ & $10 \mathrm{mg} / \mathrm{kg}$ IV every 4 weeks & PSL $1 \mathrm{mg} / \mathrm{kg} /$ day, CYC & 7 & Tapered \\
\hline
\end{tabular}

ADA, adalimumab; ANA, anakinra; AZA, azathioprine; CRP, C reactive protein; CyA, cyclosporine A; CYC, cyclophosphamide; ETN, etanercept; GC, glucocorticoid; IFX, infliximab; IV , intravenous; Ivlg, intravenous immunoglobulin; MMF, mycophenolate mofetil; MTX, methotrexate; NA, not assessed; P, primary induction; PSL, prednisolone; R, refractory/ relapsing; RTX, rituximab; SC, subcutaneous; TAC, tacrolimus; TCZ, tocilizumab. 
Contributors MA, YK and TT wrote and discussed the manuscript. All authors approved the final version of the manuscript.

Funding The authors have not declared a specific grant for this research from any funding agency in the public, commercial or not-for-profit sectors.

Competing interests MA reports no conflicts of interest relevant to this article. YK has received grants or speaker fees from AbbVie, Astellas, Ayumi, Bristol-Myers Squibb, Chugai, Eisai, Eli Lilly, Hisamitsu, Jansen, Kissei, Pfizer, Sanofi, Takeda, Tanabe-Mitsubishi and UCB. TT has received research grants or speaking fees from Astellas Pharma Inc, Bristol-Myers KK, Chugai Pharmaceutical Co, Ltd, Daiichi Sankyo Co, Ltd, Takeda Pharmaceutical Co, Ltd, Teijin Pharma Ltd, AbbVie GK, Asahi Kasei Pharma Corp, Mitsubishi Tanabe Pharma, Astra Zeneca KK, Eli Lilly Japan KK, Novartis Pharma KK, AbbVie GK, Nippon Kayaku Co Ltd, Janssen Pharmaceutical KK, Taiho Pharmaceutical Co, Ltd and Pfizer Japan Inc.

Patient and public involvement Patients and/or the public were not involved in the design, or conduct, or reporting, or dissemination plans of this research.

Patient consent for publication Not required.

Ethics approval Local ethics committee approval was not required because this study was based on published data.

Provenance and peer review Not commissioned; internally peer reviewed.

(C) Author(s) (or their employer(s)) 2020. No commercial re-use. See rights and permissions. Published by BMJ.

\section{A) Check for updates}

To cite Akiyama M, Kaneko Y, Takeuchi T. Ann Rheum Dis Epub ahead of print: [please include Day Month Year]. doi:10.1136/annrheumdis-2020-218710

Received 29 July 2020

Accepted 2 August 2020

\section{S Linked}

http://dx.doi.org/10.1136/annrheumdis-2020-218790

Ann Rheum Dis 2020:0:1-2. doi:10.1136/annrheumdis-2020-218710
ORCID iD

Mitsuhiro Akiyama http://orcid.org/0000-0001-5075-8977

\section{REFERENCES}

1 Rimar D, Alpert A, Starosvetsky E, et al. Tofacitinib for polyarteritis nodosa: a tailored therapy. Ann Rheum Dis 2016;75:2214-6.

2 Kaneko Y, Kato M, Tanaka Y, et al. Tocilizumab discontinuation after attaining remission in patients with rheumatoid arthritis who were treated with tocilizumab alone or in combination with methotrexate: results from a prospective randomised controlled study (the second year of the surprise study). Ann Rheum Dis 2018;77:1268-75.

3 Kaneko Y, Kameda H, Ikeda K, et al. Tocilizumab in patients with adult-onset still's disease refractory to glucocorticoid treatment: a randomised, double-blind, placebocontrolled phase III trial. Ann Rheum Dis 2018;77:1720-9.

4 Akiyama M, Kaneko Y, Takeuchi T. Tocilizumab in isolated polymyalgia rheumatica: a systematic literature review. Semin Arthritis Rheum 2020;50:521-5.

5 Akiyama M, Kaneko Y, Takeuchi T. Effectiveness of tocilizumab in Behcet's disease: a systematic literature review. Semin Arthritis Rheum 2020;50:797-804.

6 Kawakami T, Takeuchi S, Soma Y. Serum levels of interleukin-6 in patients with cutaneous polyarteritis nodosa. Acta Derm Venereol 2012;92:322-3.

7 Inoue N, Shimizu M, Mizuta M, et al. Successful treatment of tumor necrosis factor inhibitor-resistant cutaneous polyarteritis nodosa with tocilizumab. Pediatr Int 2020;62:753-5.

8 Krusche M, Ruffer $\mathrm{N}$, Schneider U, et al. Tocilizumab treatment for polyarteritis nodosa. Rheumatology 2020:keaa079.

9 Bodoki L, Végh E, Szekanecz Z, et al. Tocilizumab treatment in polyarteritis nodosa. Isr Med Assoc J 2019;21:560-2.

10 Saunier A, Issa N, Vandenhende M-A, et al. Treatment of polyarteritis nodosa with tocilizumab: a new therapeutic approach? RMD Open 2017;3:e000446.

11 Ostrovršnik J, Hočevar A, Lestan B, et al. Long-Term follow-up on tocilizumab treatment of $\mathrm{AA}$ amyloidosis secondary to polyarteritis nodosa. Amyloid 2016:23:260-1.

12 Watanabe K, Rajderkar DA, Modica RF. A case of polyarteritis nodosa associated with vertebral artery vasculitis treated successfully with tocilizumab and cyclophosphamide. Case Rep Pediatr 2016;2016:1-10.

13 De Virgilio A, Greco A, Magliulo G, et al. Polyarteritis nodosa: a contemporary overview. Autoimmun Rev 2016;15:564-70. 